\title{
Embedded Ubiquitous Services on Hospital Information Systems.
}

\section{AUTHOR(S):}

Kuroda, Tomohiro; Sasaki, Hiroshi; Suenaga, Takatoshi; Masuda, Yasushi; Yasumuro, Yoshihiro; Hori, Yasushi; Ohboshi, Naoki; Takemura, Tadamasa; Chihara, Kunihiro; Yoshihara, Hiroyuki

\section{CITATION:}

Kuroda, Tomohiro ... [et al]. Embedded Ubiquitous Services on Hospital Information Systems.. IEEE transactions on information technology in biomedicine: a publication of the IEEE Engineering in Medicine and Biology Society 2012, 16(6): 1216-1223

\section{ISSUE DATE:}

2012-11

\section{URL:}

http://hdl.handle.net/2433/168184

\section{RIGHT:}

(c) 2012 IEEE. Personal use of this material is permitted. Permission from IEEE must be obtained for all other uses, in any current or future media, including reprinting/republishing this material for advertising or promotional purposes, creating new collective works, for resale or redistribution to servers or lists, or reuse of any copyrighted component of this work in other works.; この論文は出版社版でありません。引用の際には出版社版をご確認ご利用ください。;This is not the published version. Please cite only the published version. 


\title{
Embedded Ubiquitous Services on Hospital Information Systems
}

\author{
Tomohiro Kuroda, Member, IEEE, Hiroshi Sasaki, Non-member, Takatoshi Suenaga, Member, IEEE, \\ Yasushi Masuda, Non-member, Yoshihiro Yasumuro, Member, IEEE, Kenta Hori, Non-member, \\ Naoki Ohboshi, Member, IEEE, Tadamasa Takemura, Non-member, Kunihiro Chihara, Senior Member, IEEE, \\ Hiroyuki Yoshihara, Non-member
}

\begin{abstract}
A Hospital Information Systems (HIS) have turned a hospital into a gigantic computer with huge computational power, huge storage and wired/wireless local area network. On the other hand, a modern medical device, such as echograph, is a computer system with several functional units connected by an internal network named a bus. Therefore, we can embed such a medical device into the HIS by simply replacing the bus with the local area network. This paper designed and developed two embedded systems, a ubiquitous echograph system and a networked digital camera. Evaluations of the developed systems clearly show that the proposed approach, embedding existing clinical systems into HIS, drastically changes productivity in the clinical field. Once a clinical system becomes a pluggable unit for a gigantic computer system, HIS, the combination of multiple embedded systems with application software designed under deep consideration about clinical processes may lead to the emergence of disruptive innovation in the clinical field.
\end{abstract}

Index Terms-Hospital Information System, Ubiquitous Computing, Echograph, Digital Camera

\section{INTRODUCTION}

$\mathbf{R}$ ECENT advancement of information and communication technologies and recent social demand for safety and efficiency of the health care services has resulted in the ongoing computerization of hospitals.

Most of hospital information systems (HIS) consist of huge computational servers which store and process electric medical records (EMR), innumerable terminals to let clinicians input or browse EMR, and information networks to connect the servers and terminals. Therefore, once a HIS is introduced, the hospital can provide ubiquitous computing services for clinicians; they can access required information anytime anywhere within the hospital.

T. Kuroda is with Kyoto University Hospital, Kyoto, 555-0025, Japan email: tomo@kuhp.kyoto-u.ac.jp.

H. Sasaki is with Kobe University

T. Suenaga is with Sendai National College of Technology

Y. Masuda is with Accense Technology Inc.

K. Hori is with Gunma Prefectural College of Health Sciences

N. Ohboshi is with Kinki University

T. Takemura is with University of Hyogo

H. Yoshihara is with Kyoto University

$\mathrm{K}$. Chihara is with Osaka Electro-Communication University

Manuscript received August 5, 2011; revised March 2, 2012 and June 1, 2012; accepted July 7, 2012; Date of publication July 26, 2012; date of current version November 16, 2012. This research was supported in part by the grantsin-aid for scientific research of japan society for the propotion of science (14208106) and inpart by the grants of the Okawa Foundation for Information and Telecommunications.
Ubiquitous computing is the term first coined by Mark Wiser [1]. Although most of social public understand the meaning of term "ubiquitous computing" as " access anytime anywhere", Weiser originally explains that "Ubiquitous computing is the method of enhancing computer use by making many computers available throughout the physical environment, but making them effectively invisible to the user." Thus, his original insight, which he tried to tell us through the technical term, must be the future to have inconspicuous support from computer system surrounding us.

Jakob E. Bardram [2] implemented Weiser's pad and wall system into hospital as a CSCW tool. Bardram's system performs as a communication board to share the on-going surgeries / treatments schedule and information of patients. Users don't care how gigantic computational power and gigantic network infrastructure support them, when they use it. They just communicate via the wall mounted touch pads. An EMR also provides such inconspicuous computational support for its users.

The same phenomena happen when we treat existing digital or medical devices. Nobody cares that a digital camera calculates blur of the obtained image to work out favorable focal length, and that an echograph illustrates a color-Doppler B-mode image from a series of ultrasound signals. Actually, most modern medical devices are computer systems.

As a medical device is a computer system, and a hospital is a gigantic computer system, the medical equipment can be embedded into the hospital. Once we embed, we may have certain advantages.

This paper prototyped several systems, an echograph and a digital camera, to be embedded into a HIS, and discusses the advantages of the approach.

\section{UBIQUITOUS ECHOGRAPH}

An echograph is one of the most popular medical imaging devices. As the composition of Siemens ACUSON P50 ultrasound system [3] clearly shows, an echograph is a computerbased system in which a sensing unit, a computation unit and a display unit is connected by internal network named a bus. Therefore, we can embed the medical device by simply replacing the bus with the information network supporting HIS.

Figure 1 shows the conceptual design of the proposed system. 


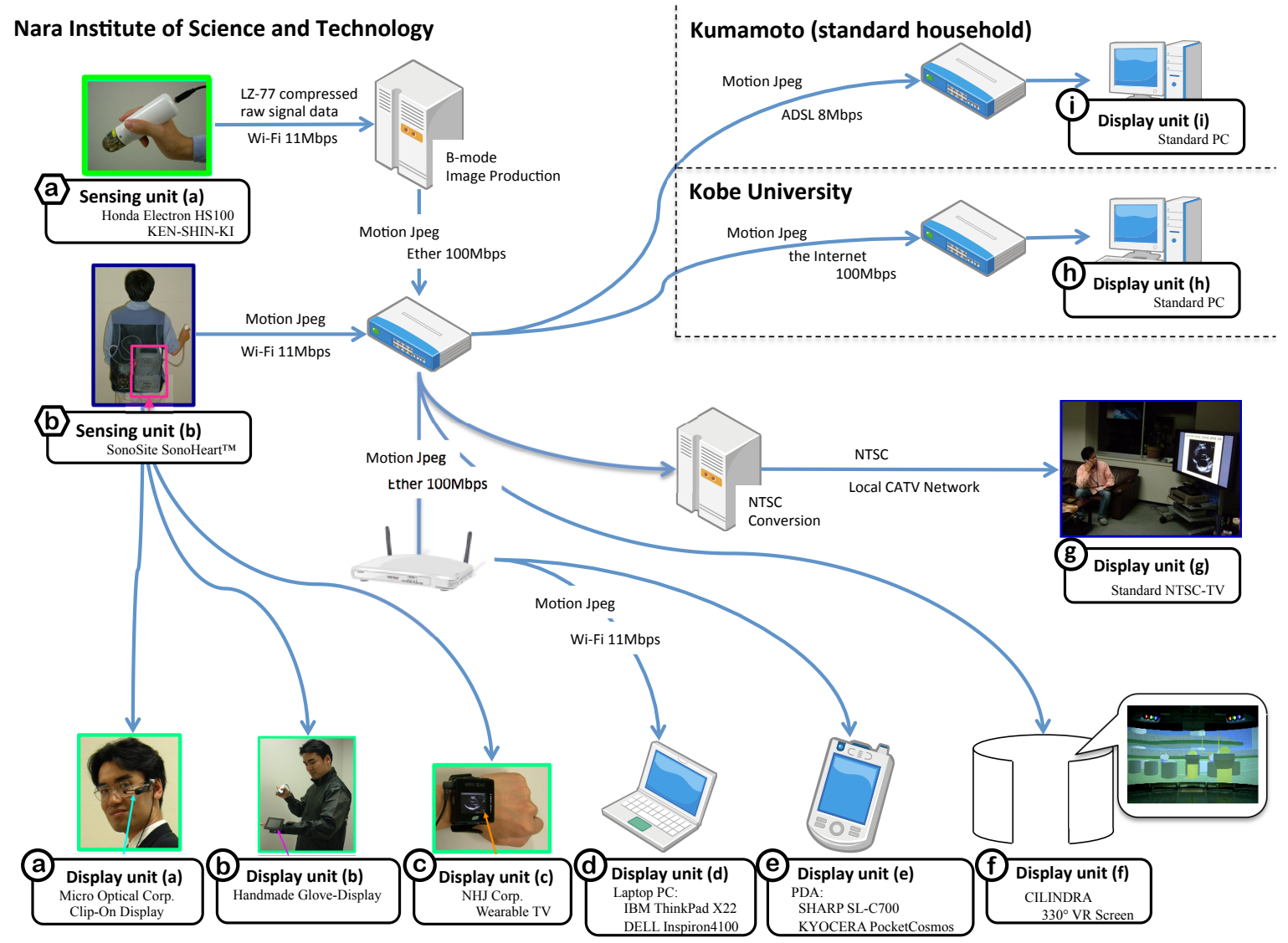

Fig. 2. The Prototype of Ubiquitous Echograph

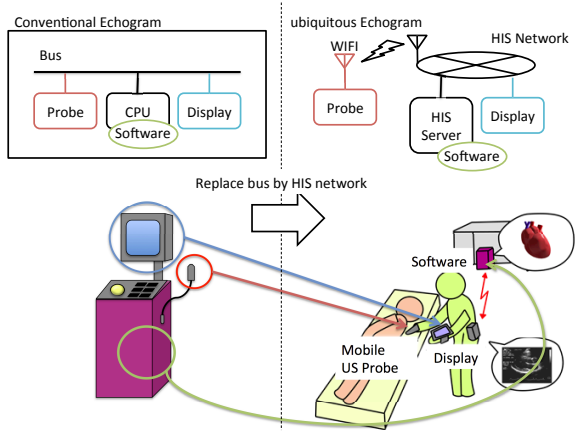

Fig. 1. Conceptual Diagram of the Ubiquitous Echograph

The system consists of a mobile ultrasound probe unit, server software, and a display. The mobile probe obtains echo signals and sends it via WiFi network. The server software installed in a HIS server re-constructs the echogram, and send it to the display. As a matter of course, the display can be any PC or PDA terminal of the HIS or any special purpose terminals such as wearable PC. Even any TV-set connected to the intra-hospital CATV network can be the display.
Once the proposed system is implemented, a medical doctor just needs to bring a mobile probe in his/her pocket to diagnose the patient with echograms at their bedside. Additionally, the hospital can easily update functions of all echograph terminals by simply upgrading the server software; even $3 \mathrm{D}$ echogram may be available when the hospital equips stereo displays. Thus, the proposed system increases mobility of the doctors (decreases load of the patients to move to diagnostic room), and decreases maintenance cost of the hospital to continuous upgrade.

The authors developed and evaluated prototype between 2003 and 2004. Figure 2 shows a construction diagram of the prototype [4].

The authors developed two different types of sensor units. The sensor unit (a) is consists of a Honda Electron HS100 named Ken-Shin-Ki, a mechanical scan 1D echo probe, connected to a Sony VGN-U71P Windows XP pocket PC via USB. It compresses resampled obtained echo signals by LZ-77 compression, and generates B-mode images by server software and sends it as a motion Jpeg image stream with quality factor of 85. The sensor unit (b) is consists of SonoSite SonoHeart Echograph connected to a handmade compact PC with Mobile Pentium III 500MHz Processor, and sends obtained image sequence by motion Jpeg. The sensor unit (b) equips three 
types of local displays, display unit (a), (b), and (c) shown in figure 2 .

The authors tested various display units, which is available under the proposed scheme. The display units (d), (e), and (f) are pseudo HIS terminals, although (f) is an unusual terminal with a SGI Onyx3800 graphics workstation with 12 MIPS R14000 CPUs and a 330 degrees immersive VR display. The display unit $(\mathrm{g})$ is a pseudo TV set at the bedside of the patient, as TV sets are located at each bedside in the most Japanese hospitals. The display unit (h) and (i) are terminals at remote site such as a huge advanced hospital.

Table I shows the obtained frame rates on each terminal. Here the local displays (a), (b) and (c) are not assesed as they are meant for diagnosis but for initial checkup of obtained echogram. The other displays are evaluated using frequency of images. The previous researches on telemedicine using echogram [5]-[7] show that only image frequency defines the legibility of echogram for the cardiologists. They could diagnose condition of mitral valve even with low quality image sequence with average peak signal-to-noise ratio (PSNR) value of $14.25 \mathrm{dD}$, if the image frequency was more than 10 frames per second (fps). As the PSNR of Motion Jpeg images with quality factor of 85 is about 30-40dB [8], [9], the legibility of echogram is reasonably evaluated by the their frequency. The result tells that the terminal (d) and (g), the standard HIS terminals, provide legible echogram stream although the display unit (e), PDA, doesn't. Additionally, the result shows that the proposed framework is even applicable for telemedicine as in the display unit (h) and (i).

\section{Networked Digital CAMERA}

A digital camera is an information system composed by three functional units, a CCD, storage, and a display, connected by a bus. Therefore, we can again embed the digital system by simply replacing the bus with LAN of HIS.

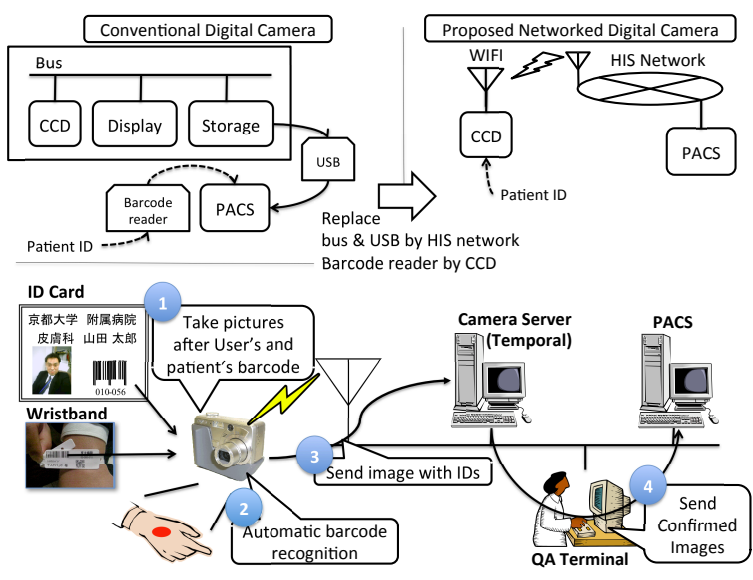

Fig. 3. Conceptual Diagram of the Networked Digital Camera System

Figure 3 shows the conceptual design of the proposed system. The system consists of a CCD and a image storage connected to HIS such as a PACS server. The image taken by the $\mathrm{CCD}$, the camera device, are sent via WiFi network and stored into the PACS.

Just as conventional medical imaging devices, photos taken by the $\mathrm{CCD}$, the camera device, are stored in the temporal server, and, after quality assurance (QA) process, confirmed photos are stored into a PACS.

In order to smoothen the process, the system should let the users input the property of the obtained photo; whose picture it is. The easiest method to identify the target patient by modern HIS is to use a barcode. Most modern HIS equips a barcode checkup system for patient safety [10]. As most barcode reading devices utilizes CCD, we can simply utilize $\mathrm{CCD}$ of the conventional digital camera to read the barcode.

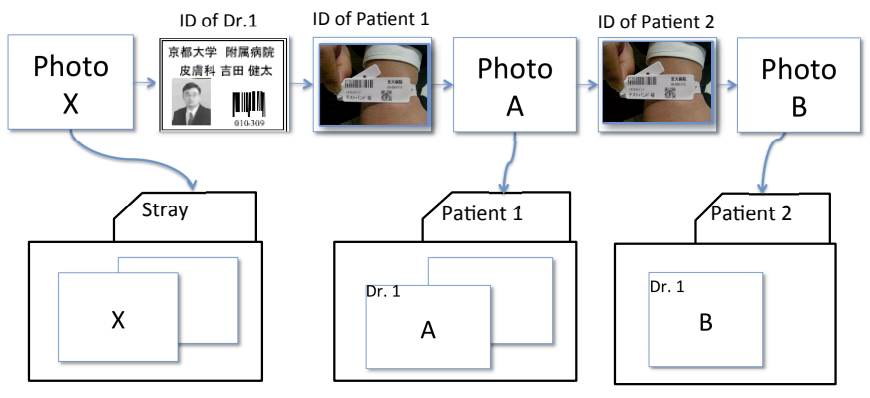

Fig. 4. ID mapping behavior of the proposed system

The proposed system works as a state machine as shown in figure 4. Once a barcode is taken, the system puts the information as a property of the following photos except for timeout. For example the photo A is marked as the patient $1 \mathrm{~s}$ image taken by the doctor 1 , and the photo $\mathrm{B}$ is marked as the patient $2 \mathrm{~s}$ image taken by the doctor 1 . If no information is given when a photo is taken, as the photo $\mathrm{X}$, then the photo is treated as a stray photo.

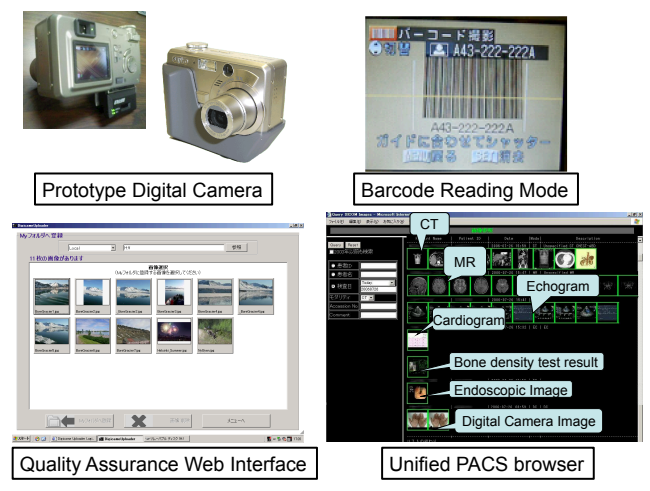

Fig. 5. The Prototype of the Networked Digital Camera

Figure 5 shows the prototype of the proposed system [11].

The prototype digital camera unit is realized by Sanyo VPCMZ3G digital camera whose firmware is modified by Sanyo Digital Technology Research Center and MELCO WLI2-CFS11 Compact Flash type WIFI card. Before taking images by digital camera, the clinician sets the camera to the barcode 
TABLE I

OBTAINED FRAME RATE ON EACH DISPLAY UNITS

\begin{tabular}{|c|c|c|c|c|c|c|}
\hline \multicolumn{2}{|c|}{ Display unit } & Image size [pixels] & Frame rate $[\mathrm{fps}]$ & Delay $(\mathrm{sec})$ & RTT (msec) & Network hop \\
\hline $\mathrm{d}$ & SGI Onyx \& Cylindra & $1024 \times 768$ & $28-30$ & & & \\
\hline $\mathrm{e}$ & IBM Thinkpad X22 & $640 \times 480$ & $28-30$ & & & \\
\hline $\mathrm{e}$ & Dell Inspiron 4100 & $640 \times 480$ & $28-30$ & & & \\
\hline $\mathrm{f}$ & Sharp Zaurus SL-C700 & $640 \times 480$ & $0.41-0.47$ & & & \\
\hline $\mathrm{f}$ & KYOCERA Pocket Cosmo & $320 \times 240$ & $2-3$ & & & \\
\hline $\mathrm{g}$ & Standard NTSC TV-set & - & $28-30$ & & & \\
\hline $\mathrm{h}$ & DELL Precision P4 3GHz & $640 \times 480$ & $28-30$ & $<1$ & 27.664 & 22 \\
\hline $\mathrm{i}$ & DELL Precision Xeon $3.2 \mathrm{GHz}$ Dual & $640 \times 480$ & $14-17$ & $1.1-1.3$ & 38.214 & 20 \\
\hline
\end{tabular}

reading mode to take the clinician's barcode and the patient's barcode as shown in upper-right of figure 5, then the clinician takes the photos of the barcode. After taking images the clinician accesses web-based QA interface, shown in lowerleft of figure 5 , developed by Tryfor Corp., which shows all the unconfirmed photos taken by the clinician. The confirmed photos are sent to GE Centricity PACS as OT image in DICOM format, any clinicians can browse the stored photos as shown in lower-right of figure 5.

The authors developed the prototype on 2004 and implemented into HIS of Kyoto University Hospital in 2005. The prototype is tested by one medical doctor and one nurse appointed by dermatology department for 1.5 years from July 2006. The prototype is used 8.5 days a month in average. The most serious problem of the prototype pointed by the subjects was that the prototype didn't have non-volatile temporal storage on its own. Therefore, if the camera run out of the battery, all the photos not sent to the server were lost. But except for that trouble, the clinicians of the dermatology department welcomed the prototype as the system dramatically reduced their photo management tasks.

Based on the experimental results with the prototype, the authors developed the commercial networked digital camera system and implemented it to the HIS of Kyoto university hospital in 2011, when Kyoto university hospital upgraded its HIS.

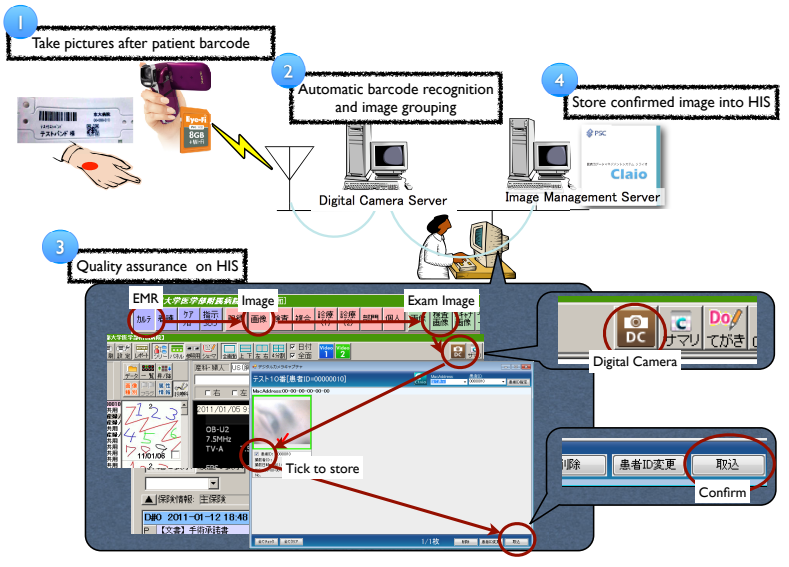

Fig. 6. The commercial Networked Digital Camera System

Figure 6 shows the developed commercial system.

The system utilizes Eye-Fi to connect digital cameras to the WiFi network. To avoid firmware modification, the camera server software developed by TriWorks Corp. checks all incoming images and recognizes the barcode. Once the camera server recognizes the patient ID by the obtained barcode, the server stores the following images to the patients temporal folder. Finally, a user browses the temporal folder and stores confirmed images via commercial image management software, Claio, developed by PSC corp. Despite from the prototype, the system doesn't request the users barcode before taking photos. Claio puts the ID of the user who confirms the photo as the user who takes the photo.

60 Sanyo Xacti DMX-CG110 cameras equipped with EyeFi and 20 additional Eye-Fi cards have been introduced to Kyoto university hospital. The cameras were distributed to all 24 inpatient wards. 17 additional cameras and two bare Eye-Fi cards were distributed to several other sections, such as ICU, outpatient ward of dermatology department etc. along requests. Most of the distributed cameras were used by the nurses as they are the main players for the patient care.

Figure 7 shows the number of taken pictures via the networked digital cameras. The system was put into operation the sixth week, in the beginning of February 2011. The figure clearly shows the number of pictures steeply increases during five months after the introduction except for the sharp decrease at the week 19 due to sharp decrease of number of patients during the holiday week at the beginning of May, which is called the Golded Week. The type of stored images were widely varied; the surgical wounds, the pressure ulcers, the rush and eruptions, the new-born babies, the max bending angles of the joints, the hand writings of patients, etc.

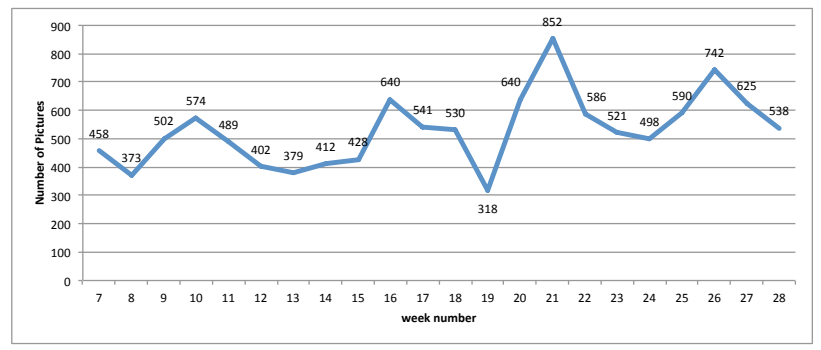

Fig. 7. The number of photos stored via the proposed system

\section{Discussions}

This paper proposed to embed the medical devices into HIS and developed two prototypes, the ubiquitous echogram and the networked digital camera. Moreover, the networked digital camera is commercialized and introduced into real clinical 
procedure within the inpatient ward of Kyoto University Hospital.

As the increase of usage shown in Figure 7 clearly shows, the commercialized networked digital camera system is warmly welcomed by clinical staffs. Actually, the director of nursing division even evaluated the camera system as "the most successful subsystem on KING5 ${ }^{1}$ ". The success firmly confirms the authors' assumption; embedding existing clinical systems into HIS drastically changes productivity in the clinical field (subjectively at least). As a fact, the minor trouble caused by the network failures or the barcode recognition error result in a lot of request from the nurses for quick repairment of the system. The steep increase of the number of photos, the utterance of the director, and the sharp increase of the claims after minor trouble, all these facts indicates that the networked digital camera system is already an indispensable tool for their daily clinical procedures.

On the other hand, the ubiquitous echograph prototype tells that even medical equipment can be embedded into HIS successfully as the standard PC terminal (display unit (c)) and standard TV set (display unit (g)) presents sufficient quality of image as shown in table I. Additionally, the prototype shows various combinations are feasible under the proposed framework.

Once a medical system such as a echograph are embedded into HIS, the system can enjoy gigantic computational power of HIS via a compact sensor unit. For example, embedded echograph may provide any complicated computation, such as cardiac output, although the prototype provide just conventional b-mode image. Additionally, once the server software is upgraded to enable more complicated computation, all the sensor units enjoy the updated function. This efficiency is one of the best fruit of the embedded system.

Despite the successful result with the prototype, the authors haven't commercialized the ubiquitous echograph prototype. The reason why the authors haven't commercialized the ubiquitous echograph is Japanese Pharmaceutical Affairs Law, which approves only united (or firmly packaged) devices not a combination of devices. According to the law, the replacement of even a single part (such as UPS or memory chip) of an approved clinical system requires new approval process. To commercialize a distributed system such as the ubiquitous echograph prototype, we need to re-organize clinical laws to fit the information age.

The recent technological advances make the ubiquitous echograph concept more realistic. The recent compact medical devices such as GE Vscan [12] enable diagnose at the bed side, and the wireless DICOM transfer function such as SonoSite NanoMaxx [13] has enables physicians to store obtained echogram to PACS servers directly. However, as the computation is performed on the compact processor unit on mobile device, users cannot enjoy the fruit of the rich computational power of HIS and the upgrade of the functions. To distribute sensing and image-generating function is indispensable to make the real fruit of the concept available.

${ }^{1} \mathrm{KING}$ is nickname of HIS of Kyoto University Hospital. Version 5 is introduced Jan. 2011
The approach to embed a medical system into HIS may fit the target medical system for other purposes. The ubiquitous echograph prototype was tested under a telemedicine scenario (display unit (h) and (i)) and the performance of the prototype was equivalent to the previous tele-diagnosis attempts [5][7]. Of course, to perform telemedicine smoothly with the ubiquitous echograph, we need to introduce additional systems to support communication between two clinicians, such as Suenaga et al [14] proposed. The clinical data transmission system cannot enable complicated multimodal communication process such as telemedicine by itself.

Quite a lot of foregoing researches on sensor networking are dedicated to remote sensing and ubiquitous health support under home environment as summarized by Pantelopulos and Bourbakis [15].

Under the scenario, various medical equipment, such as an electrocardiograph, a blood glucose meter, a saturation monitor, are connected to a sensor network over Bluetooth, Zigbee or other wireless networks for monitoring purposes [16], [17]. The potential of such wireless-ready medical equipment is maximized when it is coupled with ad-hoc sensor network, especially under disastrous condition. Some researchers developed and tested such electric triage systems [18].

As a matter of course, some foregoing researches [19]-[21] have attempted to introduce such wireless-ready devices into hospitals. Most of them just concentrate on the advancement of the sensor network infrastructure to connect various devices to conventional monitoring system available in an intensive care unit. Few [22]-[24] care about communication with an electric patient record. However, a simple conventional monitoring system is not sufficient to smoothen the clinical process; we need to consider how the wireless-ready sensor is used. The networked digital camera system is designed to smoothen QA process by the barcode recognition function.

HIS is one of the most sophisticated multidisciplinary systems. Once a medical system is re-designed to embed into HIS, the sensor connected to the sensor network become a single device unit plugged into the gigantic computer system. Therefore, just as a standard PC equips multiple sensors, a combination of multiple embedded systems may emerge in new clinical applications. Even simple positioning sensor, such as RTLS (Real-Time Location System) provided by AeroScout [25] or Ekahau [26], provides various point-of-care services as Dahl [27] discussed or context-aware services as Ho [28] presented. Information in the EPR, RTLS or even ubiquitous motion recorder proposed by Noma et al [29] provide context for the embedded system to reduce several tasks of clinical staffs as the authors discussed in [30]. Thus, embedding medical system makes HIS a truly ubiquitous computing system providing inconspicuous support as Weiser dreamed.

\section{Summary of Results}

This paper proposed to embed the medical device into the HIS, and developed two prototypes.

The authors developed the ubiquitous echograph system by replacing the bus of the echograph by the HIS network. This approach enables wide variety of setups as shown in 
figure 2, increases the mobility of doctors, and decreases the maintenance cost of the hospital to continuous upgrade. The prototype confirms that the current technology is feasible enough to enable the concept of ubiquitous echography for clinical diagnosis. Although the technology is feasible, the conventional clinical laws behind the technical innovation disable commercialization of the concept. New legal concept to support such modular medical devices is in need.

The authors developed and commercialized the networked digital camera system for clinical use by replacing the bus of the digital camera by the HIS network. The system is introduced into HIS of Kyoto University Hospital and warmly welcomed by clinical staffs. The success firmly confirms the authors' assumption: embedding existing clinical systems into HIS drastically changes productivity in the clinical field. The most important feature of the system under the comparison of the other clinical sensor-networking researches is that the unification of the device and the HIS. The system designed to smoothen QA process by the barcode recognition. To maximize fruit of the embedding devices, the deep consideration about the use case scenario is indispensable.

\section{CONCLUSION}

This paper proposed to embed medical device, a small computer system, into HIS, a gigantic computer system. The ubiquitous echograph prototype showed the potential of the idea and the networked digital camera system confirms the effectiveness of the embedded system designed under the proper consideration of clinical process. In the future, truly ubiquitous computing environment emerged by combinations of embedded medical ubiquitous services may lead to disruptive innovation in the clinical field.

\section{ACKNOWLEDGMENT}

This research is partly supported by the grans-in-aid for scientific research of japan society for the promotion of science (14208106) and grants of the Okawa foundation for information and telecommunications. The authors would like to thank Sanyo Electric, GE Healthcare, Tryfor, PSC Inc., Eye-Fi Japan, TriWorks Corp. for their precious support to prototype and to commercialize the networked digital camera system. Authors would like to thank the ulcer care team, nursing division, dermatology department and medical informatics department of Kyoto University Hospital for their continuous support to develop the networked digital camera system.

\section{REFERENCES}

[1] M. Weiser, "Some computer science issues in ubiquitous computing," Comm. ACM, vol. 36, no. 7, pp. 75-84, 1993.

[2] J. E. Bardram, "Pervasive healthcare as a scientific discipline," M. Info. Med., vol. 3, no. 47, pp. 129-142, 2008.

[3] Siemens. (2008) ACUSON P50 ultrasound system. [Online]. Available: http://www.siemens.pl/upload/images/Acuson_P50.pdf

[4] Y. Masuda, M. Naganawa, H. Sasaki, T. Suenaga, A. Yutani, Y. Yasumuro, M. Imura, Y. Manabe, O. Oshiro, and K. Chihara, "Ubiquitous tele-echograpy system: Downsized wearable ultrasound probe with distributed processors and displays," Biocybernetics Biomed. Eng., vol. 27, no. 1, pp. 247-251, 2007.
[5] T. Umeda, T. Kuroda, O. Oshiro, and K. Chihara, "A real-tome medical ultrasound image sequence transmission system with narrow integrated service digital network," Jap. J. Appl. Phys., vol. 38, no. 1-5B, pp. 34093411, 1999.

[6] T. Umeda, T. Suenaga, T. Kuroda, O. Oshiro, and K. Chihara, "A realtime telemedicine system using a medical ultrasound image sequence on a low bit-rate network," Jap. J. Appl. Phys., vol. 39, no. 1-5B, pp. 3236-3241, 2000.

[7] T. Umeda, A. Matani, O. Oshiro, and K. Chihara, "Tele-echo system: A real-time telemedicine system using medical ultarasound image sequence," Telemed. J., vol. 6, no. 1, pp. 63-67, 2000.

[8] R. Caldelli, F. Bartolini, V. Cappellini, A. Piva, and M. Barni, "A new self-recovery technique for image authentication," in Lec. N. Comp. Sci., vol. 2849, 2003, pp. 164-171.

[9] P. Hao, C. Zhang, and A. Dang, "Co-histgram and image degradaton evaluation," in Lec. N. Comp. Sci., vol. 3211, 2004, pp. 195-203.

[10] W. H. Dzik, "New technology for transfusion safety," Brit. J. Haematology, vol. 136, no. 2, pp. 181-190, 2007.

[11] T. Kuroda, T. Takemura, K. Takano, Y. Inoue, H. Miura, and H. Yoshihara, "Networked digital camera for clinical use," Jap. J. Med. Info., vol. 27 , no. 4 , pp. 355-363, 2007, japanese.

[12] G. Healthcare. (2011) Vscan let's take a look. [Online]. Available: http://vscanultrasound.gehealthcare.com/

[13] SonoSite. (2012) NanoMaxx ultrasound machine. [Online]. Available: http://www.sonosite.com/products/nanomaxx/

[14] T. Suenaga, M. Nambu, T. Kuroda, O. Oshiro, T. Tamura, and K. Chihara, "A tele-instruction system for ultrasound probe operation based on shared AR technology," in Proc. Int. Conf. IEEE Eng. Med. Bio. Soc., Istanbul, Turkey, Oct. 2001.

[15] A. Pantelopolous and N. G. Boubakis, "A survey on wearale sensorbased systems for health monitoring and prognosis," IEEE Trans. Syst., Man, Cybern. C, vol. 40, no. 1, pp. 1-12, 2010.

[16] J. S. Kim, B. O. Kim, and K. S. Park, "Development of HIHM (home integrated health monitor) for ubiquitous home healthcare," in Proc. Int. Conf. IEEE Eng. Med. Bio. Soc., Lyon, France, Aug. 2007, pp. 363-365.

[17] W. Y. Chung, Y. D. Lee, and S. J. Jung, "A wireless sensor network compatible wearable u-healthcare monitoring system using integreated ECG, accelerometer and SpO2," in Proc. Int. Conf. IEEE Eng. Med. Bio. Soc., Vancouver, Canada, Nov. 2008, pp. 1529-1532.

[18] T. Gao, T. Massey, L. Selavo, D. Crawford, B. rong Chen, K. Lorincz, V. Shanaydar, L. Hauenstein, F. Dabiri, J. Jeng, A. Chanmugam, D. White, M. Sarrafzadeh, and M. Welsh, "The advanced health and disaster aid network: A light0weight wireless medical system for triage," IEEE Trans. Biomed. Circuits Syst., vol. 1, no. 3, pp. 203-216, 2007.

[19] R. A. Rashid, M. A. Alias, and N. Fisal, "Real time medical data acquisition over wireless ad-hoc network," in Proc. Asia-Pacific Conf. App. Electr., Johor Bahru, Malaysia, Dec. 2005, pp. 20-21.

[20] H. Fariborzi and M. Moghavvemi, "Architecture of a wireless sensor network for cital signs transmission in hospital setting," in Proc. Int. Conf. Convergence Info. Tech., Gyeongju, Korea, Nov. 2007, pp. 745749.

[21] N. Damiwal, D. Ramdasi, R. Shriram, and A. Wakankar, "Wireless transfusion supervision and analysis using embedded system," in Proc. Int. Conf. Bioinfo. Biomed. Tech., Sichuan, China, Apr. 2010, pp. 17-18.

[22] P. K. K. Loh and L. Allan, "Medical informatics system with wireless sensor network-enabled for hospitals," in Proc. Int. Conf. Intel. Sensors Sensor Networks Info. Process., Merbourne, Australia, Dec. 2005, pp. 265-280.

[23] P. Frehill, D. Chambers, and C. Rotariu, "Using zigbee to integrate medical devices," in Proc. Int. Conf. IEEE Eng. Med. Bio. Soc., Lyon, France, Aug. 2007, pp. 6717-6720.

[24] P. Thongpithoonrat, P. K. McKneely, S. Gumudavelli, D. Gurkan, and F. M. Chapman, "Networking and plug-and-play of bedside medical instruments," in Proc. Int. Conf. IEEE Eng. Med. Bio. Soc., Vancouver, Canada, Aug. 2008, pp. 1514-1517.

[25] AeroScout. (2011) AeroScout tags. [Online]. Available: http://www.aeroscout.com/content/tags/

[26] Ekahau. (2011) Ekahau Wi-Fi tags operate over any Wi-Fi network. [Online]. Available: http://www.ekahau.com/products/wi-fi-tags.html

[27] Y. Dahl, "Ubiquitous computing at pont of care in hospitals: a usercentered approach," Ph.D. dissertation, Norwegian Univ. Sci. Tech., Trondheim, Norway, 2007.

[28] C. Y. Ho, "WLAN positioning for improving processes, patient care and service quality," in Proc. Wireless Cities, Oulu, Finland, Jun. 2006.

[29] H. Noma, A. Ohmura, N. Kuwabara, and K. Kogure, "Wearable seonsors for auto-event-recording on medical nursing - user study of ergonomic 
design,” in Proc. Int. Symp. Wearable Comput., Arlington, VA, Oct. 2004, pp. 8-15.

[30] T. Takemura, T. Kuroda, N. Kume, K. Okamoto, K. Hori, N. Ashida, A. Alasalmi, O. Martikainen, and H. Yoshihara, "System value analysisof multipoint distribution of realtime location system (RTLS) in hospital," J. eHealth Tech. App., vol. 6, no. 2, pp. 124-127, 2008.

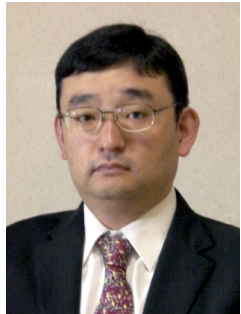

Tomohiro Kuroda (M'05) received B.E. degree in information science from Kyoto University, Japan in 1994, and M.E. and Ph.D. degree in information science from Nara Institute of Science and Technology (NAIST), Japan in 1996 and 1998, respectively.

He was with Graduate School of Information Science of NAIST from 1998 to 2001 as an assistant professor, with Department of Information Processing Science of University of Oulu, Finland in 2001 and 2006 as a visiting professor, with Department of Medical Informatics of Kyoto University Hospital, Japan from 2001 to 2006 as an lecturer, and with Graduate School of Engineering Science of Osaka University, Japan from 2007 to 2009 as an associate professor. Since 2009, he has been the vice director of Division of Medical Information Technology and Administration Planning of Kyoto University Hospital. He is also appointed as an associate professor of medical informatics of Graduate School of Medicine, and Graduate School of Informatics of Kyoto University.

Prof. Kuroda's current research interests include human interface, virtual/augmented/mixed reality, wearable/ubiquitous computing, and medical/assistive informatics. He is a member of IEEE, JSMBE, JAMI, IEICE, VRSJ, HISJ, and others.

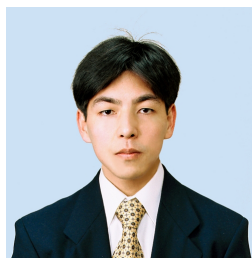

Hiroshi Sasaki received B.E. degree in information science from Kansai University, Osaka, Japan in 1996, and M.E. and Ph.D. degree in information science from NAIST in 2000 and 2003, respectively.

$\mathrm{He}$ was with Graduate School of Information Science of NAIST as a research associate from 2002 to 2004 under the 21 st Century COE Program supported by Japan Society for the Promotion of Science (JSPS). Since 2004, he has been an assistant professor of Information Science and Technology Center of Kobe University, Japan.

Dr. Sasaki's research interests include wearable/ubiquitous computing, virtual/augmented/mixed reality, human interface and science education. He is a member of VRSJ, and others.

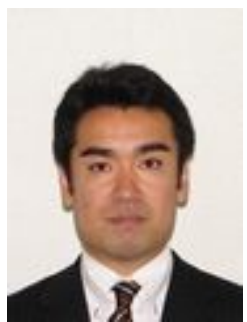

Takatoshi Suenaga (M'01) received B.E. degree in engineering from National Institution for Academic Degrees and University Evaluation, Japan in 1998, and M.E. and Ph.D. degree in information science from NAIST in 2000 and 2001, respectively.

$\mathrm{He}$ was with Telecommunication Laboratory at Universite Catholique de Louvain, Belgium from 2001 to 2003 as an assistant researcher, with Department of Medical Informatics at Kumamoto University Hospital, Japan from 2004 to 2007 as a associate professor, and with Intelligent Surgical Instruments Project at Nagoya Institute of Technology, Japan from 2008 to 2009 as an research assistant professor. Since 2009, he has been an associate professor of Department of Intelligent and Electronic Systems of Sendai National College of Technology, Japan.

Prof. Suenagas current research interests include human interface, telecommunications, embedded systems, educational information system, and welfare/assistive systems. He is a member of IEEE, JSMBE, JSME, SICE, VRSJ, and others.

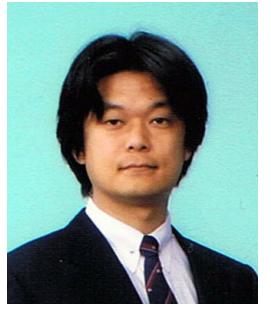

Yasushi Masuda received B.E. degree in biophysical engineering from Osaka University in 1996 and M.E. and Ph.D. degree in information science from NAIST in 1998 and 2001, respectively.

He worked in several institutes on diverse research themes, including bioinformatics, telemedicine, signal processing and ubiquitous computing. Since 2007, he has been a Director of Accense Technology Inc.

As a Director, Dr. Masuda manages R\&D projects supported by his background, such as medical information systems or distributed M2M frameworks.

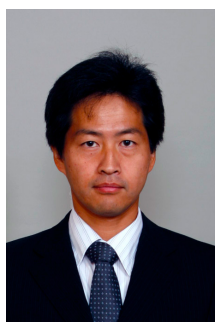

Yoshihiro Yasumuro (M'01) received B.E. degree in mechanical engineering from Osaka University in 1995, and M.E. and Ph.D. degree in information science from NAIST in 1997 and 2000, respectively.

During summer in 1999, he was a visiting scientis at Massachusetts Institute of Technology (MIT), MA. In 2000, he was a research associate at Faculty of Medicine, Osaka University under research program supported by JSPS. He was an assistant professor at Graduate School of Information Science of NAIST from 2001 to 2007. Since 2007, he joined the Faculty of Environmental and Urban Engineering at Kansai University as an associate professor. In 2010, He also joined the Department of Neuromodulation and Neurosurgery of Osaka University as a visiting associate professor.

His research interests include human-centered design and human-machine interface, using computer vision, 3-D modeling and augmented reality techniques.

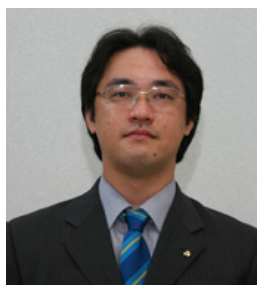

Kenta Hori received the B.E. degree in mechanical engineering from Osaka University in 1995, the M.E and Ph.D. degree from NAIST in 1997 and 2000, respectively.

From 2000 to 2003, he was a research associate with the Department of Medical Informatics of Kyoto University Hospital. From 2003 to 2005, he was a researcher with the Cell / Biodynamics Simulation Project, Kyoto University. Since 2005, he has been an assistant professor with the School of Radiological Technology, Gunma Prefectural College of Health Sciences (GCHS), Japan. Since 2009, he is also appointed as an assistant professor of the Graduate School of Radiological Technology, GCHS.

Prof. Hori's primary research interests include information systems, virtual reality, telecommunication, telemedicine, medical informatics, and medical education with information communication technologies. He is a member of VRSJ, JAMI, JTTA, JSRT, and others

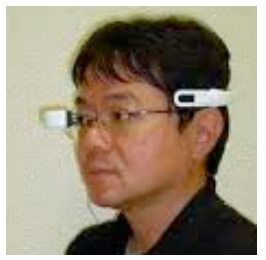

Naoki Ohboshi (M'08) received the B.E degree in communication engineering and D.D.S. from Osaka University in 1985 and 1991, respectively, and Ph.D. degree in medical science from Kyoto University in 1998.

He was with Research Center for Urban Safety and Security, Kobe University from 1997 to 1999 as an assistant professor, with Kyoto University Hospital from 2002 to 2004 as an clinical fellow, and with the School of Science and Technology, Kinki University, Osaka, Japan from 2004 to 2011 as an associate professor. Since 2011, he has been a professor of informatics at the School of Science and Technology, Kinki University.

Prof. Ohboshi's research interests include virtual/augmented/mixed reality, wearable/ubiquitous computing, and medical/assistive informatics. He is a member of the IEEE, ACM, IPSJ, VRSJ, and others. 


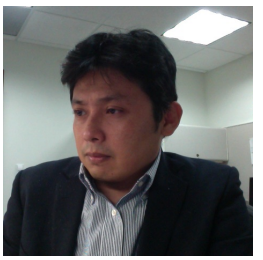

Tadamasa Takemura received B.HSc. degree in healthcare science from Osaka University in 1998 and M.HSc. and Ph.D. degree in healthcare science from Osaka University in 2000 and 2003.

He was with Department of Medical Informatics of Kyoto University Hospital from 2003 to 2007 as an assistant professor and from 2007 to 2012 as a lecturer, and with Division of Clinical Informatics of Beth Israel Deaconess Medical Center, Harvard Medical School, MA in 2012 as visiting researcher. Since 2012, he has been an associate professor of Graduate School of Applied Informatics of University of Hyogo, Kobe, Japan.

Prof. Takemuras current research interests include Natural Language Processing, knowledge engineering in Medicine, PHR, EHR, EMR, and Medical Informatics. He is a member of JSMBE, JAMI, JHM, and others.

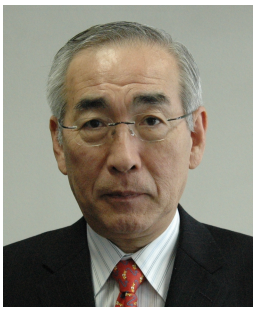

Kunihiro Chihara (M'78-SM'06) received the B.E., M.E. and Ph.D. degree in engineering science from Osaka University, Japan, in 1968, 1970 and 1973, respectively.

He was with Graduate School of Engineering Science of Osaka University as an assistant professor from 1973 to 1983 , and as an associate professor from 1983 to 1992. He was an professor of Graduate School of Information Science of NAIST from 1992 to 2011 , where he also served as the chief of information technology center (1994-1998), the chief of research center for advanced science and technology (1998-2002), the dean of graduate school of information science (2002-2007), the vice president and the director of libraries (2007-2009). He is a professor emeritus of NAIST, and has been a professor of the department of biomedical engineering of Osaka electro-communication university, Japan since 2011.

Prof. Chihara's interests include medical ultrasonic imaging, ubiquitous computing, and virtual reality. He is a fellow of IEICE, JSUM, and VRSJ, and a member of ISCIE, SSTJ, JSMBE, JSMT, JSMVR, and others.

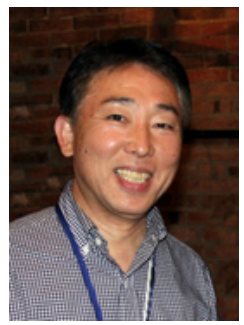

Hiroyuki Yoshihara received B.E. in engineering science from Osaka University in 1973, M.D. and $\mathrm{Ph} . \mathrm{D}$. in medical science from Miyazaki Medical College, Japan in 1980 and 1984 respectively.

After he worked as an surgeon in several hospitals, he was with Graduate School of Medical Science of Miyazaki medical college as an assistant professor from 1985 to 1995 and as an professor from 1995 to 2000. He also served as the vice director of Division of Medical Information Technology (1993-1995) and the director of Information Processing Center (19952000) of Miyazaki Medical College Hospital. He was also with Harvard University an MIT as an visiting associate professor in 1998. From 2000 to 2003, he was with Graduate School of Medicine of Kumamoto University as a professor, where he also served as director of Division of Medical Information Technology of Kumamoto University Hospital. Since 2003, He has been the director of Division of Medical Information Technology and Administration Planning of Kyoto University Hospital. He is also appointed as a professor of medical informatics of Graduate School of Medicine, and Graduate School of Informatics of Kyoto University. He has also been serving as a member of executive board of Kyoto University Hospital since 2008.

Prof. Yoshihara's current research interest includes EHR, PHR, EMR and Medical Informatics. He is a member of JAMI, JSMBE, JSSOC, and others. 